\title{
Correction: Gateway Vectors for Efficient Artificial Gene Assembly In Vitro and Expression in Yeast Saccharomyces cerevisiae
}

\author{
The PLOS ONE Staff
}

There are errors in the Results, Table 2 and Table 3, which were introduced during the typesetting process. The publisher apologizes for these errors.

There is an error in the penultimate sentence of the third paragraph of the "Construction of Yeast Gateway Vectors for One-step Gene Assembly" subsection of the Results. The correct sentence is: We compared the fluorescence produced by yEVenus-Cln $2_{\mathrm{PEST}}-\mathrm{NLS}$ and yEVenus-NLS when expressed by the constitutive promoter ADH1 (Fig 4B, C).

There is an error in Table 2, "Entry clones created in this study." Please see the corrected Table 2 here.

Table 2. Entry clones created in this study.

\begin{tabular}{lc}
\hline Plasmid & Promoter \\
pYS1 & S. cerevisiae CUP1 \\
pYS2 & S. pombe ADH1 \\
pYS3 & S. cerevisiae TEF \\
pYS6 & TetO $_{7}$-CYC1TATA \\
pYS7 & TetO $_{2}$-CYC1TATA \\
Plasmid & ORF \\
pCG32 & yEGFP \\
pDHM7 & yEGFP-CIn2PEST \\
pCG55 & yEVenus \\
pYS61 & yEVenus-NLS*1 \\
pCG98 & yEVenus-CIn2PEST-NLS \\
pCG40 & mCherry \\
pYS60 & mCherry-NLS \\
pYS19 & tTA \\
pYS20 & rtTA \\
pYS58 & AIDtTA \\
pYS57 & AIDrtTA \\
pCG72 & OsTIR1-9Myc \\
\hline
\end{tabular}

Promoter Entry clones were created with pDONR221P5-P2 and ORF Entry clones with pDONR221P1-P5r. *1 Significant cytoplasmic fluorescence was observed when overexpressed, for example, by TEF promoter. doi:10.1371/journal.pone.0150127.t001

Copyright: ( $\subset 2016$ The PLOS ONE Staff. This is an open access article distributed under the terms of the Creative Commons Attribution License, which permits unrestricted use, distribution, and reproduction in any medium, provided the original author and source are credited. 
There is an error in Table 3, "Expression plasmid vectors constructed by Gateway recombination method in this study." Please see the corrected Table 3 here.

Table 3. Expression plasmid vectors constructed by Gateway recombination method in this study.

\begin{tabular}{|c|c|c|c|c|}
\hline Plasmid & Promoter & ORF & Marker & Destination vector \\
\hline pCG52 & S. cerevisiaeTEF & mCherry & LEU2 & pDEST415TEFt7 \\
\hline pCG109 & S. pombe $A D H 1$ & mCherry-NLS & TRP1 & pDEST414TEFt7 \\
\hline pCG57 & S. cerevisiae TEF & yEVenus & LEU2 & pDEST415TEFt7 \\
\hline pRN1 & S. pombe $A D H 1$ & yEVenus-NLS & LEU2 & pDEST415TEFt7 \\
\hline pDHM57 & S. pombe $A D H 1$ & yEVenus-CIn $2_{\text {PEST }}-\mathrm{NLS}$ & LEU2 & pDEST415TEFt7 \\
\hline pCM25 & S. cerevisiae CUP1 & yEVenus-CIn2PEST-NLS & LEU2 & pDEST415TEFt7 \\
\hline pCG87 & TetO $_{7}$-CYC1TATA & mCherry-NLS & TRP1 & pDEST414TEFt7 \\
\hline pCG103 & TetO $_{7}-\mathrm{CYC}_{1 \mathrm{TATA}}$ & yEVenus-CIn $2_{\text {PEST }}$-NLS & TRP1 & pDEST414TEFt7 \\
\hline pCM20 & TetO $_{7}-\mathrm{CYC1TATA}$ & yEVenus-CIn $2_{\text {PEST }}-\mathrm{NLS}$ & LEU2 & pDEST415TEFt7 \\
\hline pCG84 & S. pombe ADH1 & tTA & HIS3 & pDEST413TEFt7 \\
\hline pCG85 & S. pombe $A D H 1$ & rtTA & HIS3 & pDEST413TEFt7 \\
\hline pDHM19 & S. pombe $A D H 1$ & AIDtTA & HIS3 & pDEST413TEFt7 \\
\hline pDHM20 & S. pombe $A D H 1$ & AIDrtTA & HIS3 & pDEST413TEFt7 \\
\hline pCG112*1 & S. cerevisiae TEF & tTA & HIS3 & pDEST413TEFt7 \\
\hline pCG113 & S. cerevisiae TEF & rtTA & HIS3 & pDEST413TEFt7 \\
\hline pCG106*1 & S. cerevisiae TEF & AIDtTA & HIS3 & pDEST413TEFt7 \\
\hline pCG107 & S. cerevisiae TEF & AIDrtTA & HIS3 & pDEST413TEFt7 \\
\hline pMM6*2 & S. cerevisiaeTEF & AIDrtTA & URA3, MET15 & pDEST375 \\
\hline pCG81 & S. pombe $A D H 1$ & OsTIR1-9Myc & URA3 & pDEST416TEFt7 \\
\hline
\end{tabular}

${ }^{* 1}$ Yeast cells with these expression vectors showed poor growth with an unknown reason.

$*^{2}$ Integration vector.

doi:10.1371/journal.pone.0150127.t002

\section{Reference}

1. Giuraniuc CV, MacPherson M, Saka Y (2013) Gateway Vectors for Efficient Artificial Gene Assembly In Vitro and Expression in Yeast Saccharomyces cerevisiae. PLoS ONE 8(5): e64419. doi:10.1371/ journal.pone.0064419 PMID: 23675537 